\title{
Die Rückfallsgrippe.
}

(Chronische Influenza. Rezidivierende fragmentarische Influenza. Afebrile Nerveninfluenza.)

Von Priv.-Doz. Dr. med. et phil. Willy Hellpach in Karlsruhe.

Die Charakterveränderung, der die großen Seuchen mit ihrer Dauer anheimfallen, ist eines der interessantesten und vielleicht noch viel zu wenig durchforschten Probleme der Pathologie. Regelmäßig handelt es sich dabei um eine fortschreitende Abschwächung der augenfälligen Wirkungsstärke des infektiösen Giftes, die zum völligen Verschwinden der Seuche, ja zu ihrem Vergessenwerden, ihrem Wegfall aus dem Lexikon der Krankheitsnamen führen kann - einem Verschwinden, dem dann nach Jahrzehnten freilich eine Art verjüngter Wiederkehr zu folgen pflegt - oder in anderen Fällen (für lange Zeit wenigstenş) eine Art stationären Zustandes mit milderen, von der früheren Gestalt wesentlich abweichenden Eigenschaften bewirkt.: Die Influenza war verschollen ge- 
wesen, als sie 1889 mit ungeheurer Vehemenz hervorbrach; die Syphilis hat sich gegenüber ihrem Charakter in früheren Jahrhunderten wesentlich benigner gestaltet, scheint aber seit Jahrzelinten im ganzen gleichartig zu bleiben; vielleicht liegt freilich nur eine so langsame Wandlung vor, daß sie erst in größeren Zeitabschnitten deutlich wird.

$\mathrm{Ob}$ es sich nun bei derartigen Veränderungen der Giftwirkung um eine Umbildung der Giftträger oder um eine solche des befallenen Organismus im Sinne der Immunisierung handelt, oder um ein Zusammenwirken beider Faktoren, ist eine keineswegs geklärte Frage der Epidemiologie. Unverkennbar nur ist in manchen Beispielen die Erscheinung, daß die Milderung des Krankheitscharakters mit einerVerschiebung von der akuten zur chronischen Erscheinungsform und insbesondere wieder mit zunehmender Bevorzugung der chronischen Einnistung i m Nervensyste $m$ Hand in Hand geht. Das trifft zu auf die Syphilis, deren maligne Form bei uns fast ganz verschwunden, deren Lokalisation in den nervösen Zentralorganen, besonders als ,metasyphilitische“ Erkrankung, aber zweifellos häufiger geworden ist. Allerdings hat man versucht, sich dies mit der größeren Widerstandsschwäche des modernen Kulturmenschen in puncto Nervensystem, namentlich Gehirn, zu erklären; und durch die Edingersche Hypothese der ,Aufbrauchskrankheiten" scheint eine solche Erklärung besonders nahe-. gelegt zu werden. Indessen, diese Hypothese einmal als erwiesenes Gesetz angenommen, handelt es sich bei dem, was sie zu erklären sich anheischig macht, doch garnicht so sehr (wie der etwas schiefe Name zunächst vermuten läßt) um die Bestimnung ganzer Krankheitsformen durch Aufbrauch, sondern lediglich um die Gruppierung der Symptome, ihres zeitlichen Auftretens und ihrer Schwere nach der Inanspruchnahme der entsprechenden Organe (Ptosis durch Uebernächtigkeit, Verteilung der befallenen Muskeln bei Bleilähmung nach der beruflichen Beanspruchung, Ataxie nach Märschen u. dgl.), die zugrundeliegende Krankheit aber fließt aus einer davon gesonderten Quelle (Tabes metasyphilitisch etc.). Man wird nun kaum behaupten wollen, daß die Paralyse z. B. nur ein neues ,Symptom" sei; wenn sie innerhalb gewisser Kulturen, wie es scheint, nie vorkommt, trotz verbreiteter Syphilis, innerhalb anderer sich dagegen als metasyphilitisches Phänomen einstellt, so dürfte es sehr schwer sein, bloß aus der stärkeren Hirnbeanspruchung, die diese letzteren Kulturen mit sich bringen, eine Neuheit von solcher Tragweite herzuleiten. Edinger will zwar soweit gehen, wenn ich ihn recht verstehe, aber er wird nicht viele bis dahin mit sich fortreißen, so bestechend im Bereiche der Sy m p to men erklärung sein Deutungsversuch ist. Mindestens ebenso nahe liegt doch die andere Annahme, daß das infektiöse Gift sich geändert habe, sei es in spontaner Fortentwicklung, sei es in Wechselwirkung mit der Immunisierung, in die es selber allmählich die von ihm befallene Organismenwelt versetzt hat; und diese Annahme wird wahrscheinlicher, wenn sich zeigt, daß dieses Gift für andere Gewebe seine Malignität, ja daß es ïberhaupt seine akute Malignität gemildert hat: das kann man, alles moderne ,Nervenmenschentum" zugegeben, doch ganz unmöglich daraus ableiten, daß etwa früher die Menschen die anderen Organsysteme so viel mehr abgenutzt hätten. Wenn einstens die sekundäre Syphilis so viel gefürchteter verlief, heute aber an der ganzen Krankheit das Bedrohlichste ihre chronischen tertiären und metasyphilitischen Prozesse im Nervengewebe sind, so sehe ich keinen plausiblen Weg, Aufbrauchsunterschiede dafür verantwortlich zu machen, wenigstens nicht in einigermaßen hinreichendem Umfange.

Ein ganz ähnlicher Prozeß aber, wie ihn die Syphilis im Laufe von Jahrhunderten uns zeigt, scheint bei der Influenza im Laufe von zwei Jahrzehnten sich entfaltet $\mathrm{zu}$ haben; si parva licet componere magnis. Wo sind doch die schweren Fälle der Epidemie von 1889 und auch der von 1896? Die massenhaften Herztode, Pneumonien, Enzephalitiden? Ihre Ziffer hat auch prozentual ganz gewaltig abgenommen. Statt dessen aber quält sich ein sehr erheblicher Bruchteil der Menschen nunmehr mit höchst merkwürdigen chronischen Belästigungen, die ihren Zusammenhang mit der Influenza nur noch durch gelegentliche akute Exzedentien ver- raten, sowie dadurch, daß sie bei sorgfältiger Betrachtung die Hauptsymptome des ,Typus nervosus" der vehementen Seuche frïherer Jahre in gemilderter und zeitlich protrahierter Form erkennen lassen. Es ist leicht begreiflich, daß diese neue Form noch wenig medizinische Wïrdigung gefunden hat: Monographien und Kasuistiken liefern zumeist die Leiter und Assistenten unserer Krankenhäuser. Dieser Ort wird aber von denen fast nie aufgesucht, die von chronisierten Influenzasymptomen geplagt sind; selbst den Arzt nehmen diese Kranken oft nicht in Anspruch. Wir werden sehen, daß mancher von ihnen durch das eigenartige Bild sich zum Neurologen treiben läßt, wo er wohl oft genug die Etikette „Hypochonder", ,Neurastheniker" u. dgl. empfängt, mit der übrigens der eventuell konsultierte Haus- oder Kassenarzt eher noch freigiebiger zu sein pflegt. Immerhin ist seit Jahren gelegentlich auf einzelne Sypnptome solcher Art, anı nachdrücklichsten von Felix Franke in Braunschweig, hingewiesen worden; er hat neuerdings eine zusammenfassende Betrachtung darïber veröffentlicht. ${ }^{1}$ )

Man wird bald finden, daß die folgende Darstellung von der Frankes beträchtlich abweicht; trotzdem mag zunächst einmal das Gemeinsame hervorgehoben werden. Unter allen Umständen handelt es sich darum, daß Menschen wochenund monatelang von merkwürdig wechselnden Symptomen im Bereiche der Nervenfunktion (vorwiegend wenigstens) belästigt, geängstigt, benachteiligt werden, daß ein plötzlicher Ausbruch katarrhalischer Erkrankung, mit geringem oder ganz ohne Fieber, aber heftigen Katarrherscheinungen (anginöser, koryzaler, bronchitischer, konjunktivitischer, stomatitischer und ähnlicher Art) jene nervösen , löst" und tage-, auch wohl wochenlange Befreiung von ihnen schafft, bis sie in der alten oder in neuer Gruppierung wiederkehren. Dieses Spiel, das jahrelang währen kann und das dem klinischen Auge wie eine immer rezidivierende, aber sehr fragmentarische Influenza mit ungeheuer lang hingedehnten Prodromen erscheinen $\mathrm{muß,}$ möchte ich ebendarumals chronische, rezidivierende, fragmentarische Influenza, oder in verdeutschender $\mathrm{Ab}$ kürzung dieses terminologischen Ungeheuers, kurzweg als Rückfallsgrippe bezeichnen.

Ich lasse nun, entsprechend meiner Hauptabsicht, eine möglichst erschöpfende Schilderung der chronischen Nervensy mptome, die das Leiden charakterisieren, folgen:

I. Symptome. 1. Stimmung. Veränderungen der Stimmung in pejus begleiten die hier geschilderten. Krankheitszustände zunächst natürlich so weit, wie sie mit allem Kranksein (von pathologischer Euphorie abgesehen) verknüpft zu sein pflegen. Auffällig ist aber bei der Rückfallsgrippe ihr vielfach in die Augen springendes Mißverhältnis zu der Intensität der ïbrigen Symptome. Namentlich die Depression (bald in gereizter, bald in weinerlicher, bald in ratloser und ähnlicher Nuancierung) kann Grade erreichen, die den Kranken selber unfaßlich sind. Wir sehen natïrlich von der Depriemiertheit, die ein monatelang ewig wiederkehrendes Krankwerden als seelische Reaktion auslöst, sowie von den hypochondrischen Grillen, die sich daran gleichsam natürlich anschließen, ab. Aehnlich wie beim Schnupfen und bei der akuten Influenza leitet eine Depression oft prodromal die Anfälle der Rückfallsgrippe ein, ihre Heftigkeit steht aber, wie bemerkt, meistens und gerade auch bei keineswegs neuropathischen Patienten außer Verhältnis zu dem, was nachfolgt. Einem ganz leichten, mit mäßiger Nasensekretion und ein paar anderen Symptomen (z. B. neuralgischen, rheumatischen etc.) einhergehenden Anfall kann durch eine tief deprimierte stundenlange, selbst tagelange Verstimmung mit starker ",Geladenheit" oder völliger Verzagtheit präludiert werden. Mit dem Ausbrechen der genannten katarrhalischen Anfallserscheinungen pflegt die Verstimmung entweder ,lytisch“ oder wohl noch öfter in raschem Umschwung sich zu lösen. Der Zusammenhang der Depression mit dem nachfolgenden Anfall kann so typisch sein, daß die Patienten ihre unmotiviert

1) Beiheft 10, Jahrgang 1909 der Medizinischen Klinik: „Ueber chronische Influenza“. 
sie befallende Verstimmung schließlich als Vorboten des Anfalls richtig deuten und dann auch leichter ertragen.

2. Geistige Arbeitsfähigkeit. Sie ist, auch wo keine Depression sie ungünstig beeinflußt, sehr oft stark vermindert. Die Herabsetzung verläuft im ganzen unter dem früher häufig geschilderten Bilde der "Influenza-Neurasthenie" milderer Form. Es besteht Arbeitsunlust, Gehemmtheit, Schläfrigkeitsgefühl, Abspannung, Eingenommenheit des Kopfes in allen Varianten, Mangel an Konzentrationskraft, äußerst rasche Ermüdbarkeit, subjektives Gefühl der Gedächtnisschwäche. Alles das kann sich aber iiber den Anfall hinaus erstrecken, die Patienten fühlen sich auch in den von speziellen Symptomen freien Intervallen, ,nicht auf der Höhe". Vor und während der Anfälle können allerdings beträchtliche Steigerungen eintreten. Die antineurasthenischen Mittel versagen oft gänzlich, statt dessen tritt dann ganz unerwartet eine rasche Besserung der geistigen Hinfälligkeit bis zum völligen Verschwinden ein - ohne besondere "Schonung" oder „Ausspannung“. Daß bei langer Dauer die Patienten durch das Gefïhl des Nachlassens ihrer Leistungsfähigkeit sehr deprimiert werden und dann auf diese Weise ein Circulus vitiosus sich herausbildet, der die Arbeitsfähigkeit noch mehr beeinträchtigt, ist begreiflich. Als besonderes, auffallend oft bemerkliches Symptom in Bilde der herabgesetzten Leistung mag erwähnt sein:

3. Eine Art Paraphasie, nämlich die Neigung sich zu versprechen, über Konsonanten zu stolpern, Wörter schwer zu finden - ähnlich wie sie bei der Neurasthenie vorkommt.

Ein Herr kam in großer Angst über dieses Symptom zu mir, er meinte am Vorabend der Paralyse zu stehen, zumal auch die später zu erwähnenden fibrillären Zuckungen und spastischen Symptome ihn erschreckten. Mit dem Ausbruch eines vehementen "Schnupfens" löste sich alles das in Wohlgefallen auf.

Paradigmatisches Silbenstolpern wird aber (wie bei der Neurasthenie) so auch hier wohl niemals beobachtet.

4. Schwindel. Er ist überaus häufig, ähnlich der Depression, vielleicht noch häufiger und in allen Intensitäten zu finden. Sein Erscheinen ist hauptsächlich prodromal. Er kann so konstant sein, daß fortwährend der Boden, die Tischplatte und dergleichen sich hebt und senkt, er kann aber auch in einzelnen heftigen Attacken eintreten, kann mehr vor den Augen oder mehr im Lagegefühl sich geltend machen. In Verbindung mit der Herabsetzung der geistigen Arbeitsfähigkeit, dem Kopfdruck und den noch zll erwähnenden Augenstörungen erzeugt er bei den Patienten nicht selten ein Gesamtgefühl von unsicherer Benommenheit, das sehr peinlich ist. „Ich lauf herum wie dämlich", beschrieb mir ein Kranker den Zustand. Der Anfall pflegt den Schwindel zu beseitigen, wenigstens die stärkeren Grade. Zu beachten ist, da $\beta$ die Schwindelattacken schein bar verursacht werden können durch Magenbelästigung, angestrengte Augenkonvergenz $u$. dgl., obwohl früher Magenschwindel, Augenschwindel etc. dem Patienten unbekannt waren. Der Verlauf zeigt dann, daß diese Faktoren nur eine auslösende Rolle spielen.

5. Beklemmung. Sie ist oft ein Teilsymptom der Depression, kommt aber auch ohne wesentliche Stimmungsveränderung als mehr physisch empfundene Oppression auf der Brust vor. Das tiefe Einatmen ist erschwert, die Brust ist wie zusammengepreßt, zu eng. Manchmal scheint ein Zusammenhang mit dyspeptischen Symptomen zu bestehen. Niesen, häufig das Signal des einsetzenden Anfalls, scheint Erleichterung bringen zu können.

6. Alkoholintoleranz. Sie ist in einzelnen Fällen ganz frappant als prodromales, auch gelegentlich in den Anfall hineinreichendes Symptom zu beobachten. Kleine Dosen, sonst kaum von nennenswerter Wirkung, rufen Gereiztheit oder starke motorische Erregtheit (Unruhe, Zittern, „Geladenheit") oder Schwindel, Betrunkenheitsgefühl und ähnliches hervor; ziehen wohl auch ,Katzenjammer" von unverhältnismäßiger Stärke nach sich.

Wenn (beim selben Patienten) zuweilen das Umgekehrte beobachtet wird, nämlich außerordentlich günstige Wirkung des Alkoholgenusses (unter Umständen sogar gerade eines alkoholischen Exzesses) am folgenden Tage, so erklärt sich das hinreichend aus den neuereren Feststellungen über das Ver- hältnis von Alkohol und Infektion überhaupt. Danach gibt es eine Initialphase der Infektion, in der der Alkohol offenbar antitoxisch wirkt, während späterhin diese Wirkung entfällt und die Herabsetzung der allgemeinen Widerstandsfähigkeit der Gewebe durch den Alkohol vorherrschend in Erscheinung tritt. Die Erfahrung der einen, daß man durch große Dosen von Alkohol ,Katarrhe“" kupieren kann, und die der anderen, daß man mit solchen Zuständen am schnellsten fertig wird bei abstinentem Verhalten, sind also nur scheinbar entgegengesetzt. Das gilt alles auch für die Rückfallsgrippe. $\mathrm{Zu}$ beachten wäre bei dieser Erkrankung freilich noch, da $\beta$ der Alkohol die katarrhalische Sekretion steigert (man denke an die Bronchial- und Rachenkatarrhe der Trinker) und vielleicht auch dadurch Erleichterung des Allgemeinbefindens schaffen kann, indem eben erfahrungsgemä $\beta$ jedes rasche Steigen der manifesten katarrhalischen Erscheinungen - „der Anfall“ - als wohltätige Entlastung von den unbestimmten Prodromalsymptomen empfunden wird. Dann würde also der Alkohol nicht durch seine antitoxischen Eigenschaften, sondern durchs Gegenteil günstig wirken. Wobei allerdings wieder das Fragezeichen bleibt, ob nicht der manifeste Ausbruch einer infektiösen Erkrankung iiberhaupt eine Art "Heilungsproze $B^{\circ}$ des Organismus darstellt (in dem die antitoxischen Elemente freilich unterliegen können). Diese Probleme der Infektionspathologie sind fa noch viel zu wenig geklärt, un mehr als Vermutungen zuzulassen.

Intoleranz wurde bei der Rïckfallsgrippe gelegentlich auch gegen andere Gifte, z. B. Nikotin und Kaffee, angegeben.

7. Schlafstörungen. Die häufigste Form ist unruhiger, traumreicher, nicht erquickender Schlaf im Prodromalstadium des katarrhalischen Anfalls. Angstträume, Beklemmungen, selbst Aufschrecken, Aufschreien, Aufsitzen und ähnliches kommen bei Leuten vor, denen sonst derartige Erlebnisse völlig fremd sind. Eine Dame prognostizierte aus solchen Vorkommnissen bei ihrem Gatten schon immer den heranziehenden "Schnupfen". Während des Anfalls habe ich wiederholt eine andere Schlafstörung beobachten können, die von septischen Infektionen her mir bekannt war: das frühe Erwachen nach gutem Einschlafen und mehrstündigem Schlaf, verbunden mit einer Illusion von Ausgeschlafenheit, die beim Aufstehen aber bald einer lähmenden Abgeschlagenheit weicht.

In einem Fall von Empyem der Highmorshöhle, der mich wegen der neuralgiformen Symptome aufsuchte, schwand diese seit Jahr und Tag vorhanden gewesene Störung mit einem Schlage nach der Punktion des Empyems, kehrte im Rezidiv wieder und schwand definitiv mit der endgültigen Heilung. Das Gleiche ist mir bei Rückfallgrippe mehrfach berichtet worden, einmal führte sogar lediglich diese Art Schlafstörung den Patienten zu mir. Sie verband sich in diesem Falle übrigens mit plötzlich einsetzender gı oßer Schläfrigkeit zu ganz unnormalen Tageszeiten, ähnlich wie sie bei der Neurasthenie beobachtet wird.

Ein Zusammenwirken von Alkoholintoleranz mit Schlafstörung kommt in der Art häufig vor, daß sonst als Schlafbeförderer genommene Alkoholdosen erregend und damit schlafhindernd wirken oder sehr frühes Erwachen provozieren. (Das frühe Erwachen wird übrigens von recht vielen Menschen als Effekt alkoholischer Exzesse am Vorabend wahrgenommen, wie es fa außerdem ein Symptom des Alters und der Arteriosklerose ist.)

8. Störungen der Sexualität. Sie können ähnlich der Depression und dem Schwindel zu den klassischen Vorboten des Anfalls gehören, wie übrigens bei allen Infektionen. Ein sehr leistungsfähiger Herr weissagte seinen "Katarrh" regelmäßig aus dem Auftreten von Ejaculatio praecox, und zwar immer richtig. Es kommen aber alle Formen und Kombinationen vor. Am häufigsten dürfte Mangel an Libido, manchmal in Verbindung mit gehäuften spontanen Erektionen oder sogar nächtlichen Pollutionen sein. Aber auch Versagen der Potenz, namentlich mitten in actu, Ejaculatio praecox bei ungenügender Erektion, mangelnder Orgasmus $u$. a. werden beschrieben. Alles das ist als Ausdruck einer toxisch gesteigerten Erregbarkeit der Zentralorgane leicht verständlich. Es kann mit dem Anfall schwinden, aber auch ihn überdauern bis zum Abklingen des Anfalls. Die Plötzlichkeit und Stärke der. Erscheinungen steht auch dabei wieder meist außer Ver- 
hältnis zu der Geringfügigkeit des eigentlichen Krankheitsbildes. - Auf weiblicher Seite wurde einmal abnorm gesteigerte sexuelle Erregbarkeit in Prodromalstadium beobachtet.

9. Verdauungsstörungen. Die weitaus häufigste, die wir ebenfalls zu den ,klassischen" Prodromen rechnen müssen, ist die spastische Verstopfung. Das Auftreten bleistiftförmiger Stuhlmassen, die nur unter starker Anstrengung entleert werden können, kündigt nicht wenigen Menschen an sich schon katarrhalische, schnupfenartige Erkrankungen an. Die spastische Diarrhoe ist entschieden in diesem Zusammenhang seltener, allerdings muß ish bemerken, daß sie neuerdings im Bilde der Rückfallsgrippe in den Vordergrund zu treten scheint. Häufig kombiniert sich beides, sogar während einer Entleerung: nachdem ein paar spastisch geformte Gebilde herausgepreßt sind, folgt plötzlich ganz dünnbreiiger Stuhl, manchmal unter Kolikerscheinungen, dann wieder harte, geformte Massen. Nach der Fntleerung bleibt stundenlang ein unsicheres, unruhiges Gefühl zurück; Tenesmus ist sehr häufig. Die Symptonie sind sprunghaft, bleiben tagelang fort, kehren plötzlich wieder und zeigen einen charakteristischen Zusammenhang weder mit Diätexzessen noch mit seelischer Erregung. Mit dem Ausbruch des katarrhalischen Anfalles sind sie wie mit einem Schlage behoben, um dann eventuell wiederzukehren. Die Stuhlfärbung scheint oft auffallend wechselnd, von einer Entleerung bis zur nächsten zwischen hellbraun und ganz dunke] schwankend zu sein. Das Auftreten des Stuhldranges ist oft kolikartig, äußerst vehement. Die Flatus sind reichlich und übelriechend, selbst nach blandester Diät, und manchmal in ihrem Fortwandern überaus schmerzhaft.

Vom oberen Teil des Verdauungskanals her wäre Appetitlosigkeit mit widerlichem Geschmack in Munde, belegter Zunge, Aufstoßen, Sodbrennen, Druckgefühl nach dem Essen, Heißhunger, Uebelkeit, Widerwille gegen Speisen, kurz der ganze Komplex der sonst nervös-dvspeptischen Störungen zu nennen. Erbrechen habe ich nicht gesehen.

10. Schmerzen. Sie sind für das Bild der Rückfallsgrippe so charakteristisch, daß sie als klassisches Symptom angesprochen werden müssen. Thre häufigsten beiden Varianten sind die akralgische und die neuralgische Form.

Unter Akralgien wollen wir Schmerzhaftigkeit aller möglichen Endglieder, Vorsprünge u. dgl. mehr verstehen. Sie sind für: unsere Erkrankung geradezu typisch (Frankes ,Influenzaknie" ist nur eine bestimmte Form davon). Cristae, Condyli, Tubera, Gelenkenden, Capitula und was derlei mehr ist: sie alle können tage-, wochen-, monatelang der umschriebene Sitz einer intensiven, manchmal spontanen, manchmal nur auf Druck (dann aber oft schon auf leise Berührung) fühlbaren Schmerzhaftigkeit sein. Der Schmerzhaftigkeit entspricht keinerlei objektiver Befund: keine Schwellung, keine Auflagerung, keine Rauhigkeit, keine Deformität. Sie kommt meist wie ,angeflogen", besteht eine Weile, ist dann plötzlich verschwunden und taucht irgendwoanders wieder auf. Die Patienten zerbrechen sich häufig den Kopf, wo sie sich ,,angeschlagen" oder sonstwie traumatisch geschädigt haben mögen, da sie sich nur so den seltsamen Schmerz erklären können. Reißend ist der Schmerz fast nie, immer so gut wie konstant. Sein urplötzliches Weichen mit dem Ausbruch der katarrhalischen Sekretion läßst seine wirkliche Ursache erkennen.

Neuralgische Schmerzen kommen nicht minder häufig vor, bald als echte, ortsständige, bald als vagierende Neuralgien. Supraorbital-, Intrakostal- und Ischiasfornien scheinen mir vorzuwiegen. Der Charakter der Schmerzanfälle ist oft verwaschen. So habe ich wiederholt Fälle gesehen, die wegen „Stirnneuralgie" zu mir kamen und ein merkwürdiges Mischungsbild zwischen der echten Supraorbitalneuralgie und dem Schmerzanfall bei Stirnhöhlenkatarrh darboten. Nicht selten stellen sich die Paroxysmen um bestimmte Tagesstunden ein; die Intensität kann sehr groß sein, ist wohl aber im Durchschnitt milder als die der echten Neuralgie. Die rhinologische Untersuchung verlief regelmäßig negativ, soweit die Stirnhöhle in Frage kam. Bei den Ischiasformen fehlt sehr häufig das Ischiasphänomen, auch hier ist das ganze Bild weniger klassisch; der Schmerz springt nicht selten ganz plötzlich auf die andere
Seite über. Häufig sind auch neuralgiforme Beschwerden im Brachialgebiet, nicht selten kombiniert mit schulterrheumatischen Symptomen oder rheumatischem ,Zupfen" in den kleinen Gelenken der Finger. Alle neuralgischen Schmerzformen können ausgesprochen schießend, „, lanzinierend" auftreten, d. h. es erfolgt immer nur nach Stunden oder Minuten ein einziger heftiger Schmerzstich, dessen Intensität dann freilich nicht selten so groß ist, daß die Patienten Gegenstände. fallen lassen (bei Armschmerz) oder zusammenknicken (bei Beinschmerz). Okzipitale Schmerzen sird mir nicht sehr oft vorgekommen; einmal allerdings sehr heftig in Mischung mit akralgischer Schmerzhaftigkeit der obersten drei Wirbelfortsätze.

Aeußerst charakteristisch finde ich die neuralgiformen, manchmal aber auch akralgiformen Schmerzen in inneren Organen. Die Reihe eröffnet der Dickdarm. Ganz bestimmte, umschriebene Partien desselben, wobei die Ileozökalgegend benerkenswerterweise keine Prädilektionsrolle zu spielen scheint, können tage-, ja wochenlang spontan oder auf leisesten Druck intensiv schmerzempfindlich sein. Auch die Durchwanderung von Gasen durch diese Partien wird dann als eminent schnierzhaft verspürt. Eines Tages ist der Schmerz fort oder sitzt anderswo. Die Flexura sigmoidea und die Partien vor ihr scheinen am meisten ergriffen zu werden. Dann käme der Magen. „Magenkrämpfe“, d. h. Magenschmerzparoxysmen sind überaus häufig und variieren von leisester Schmerzhaftigkeit nach dem Essen oder nach kühlem Trinken bis zu den unerträgliuhsten Attacken. Kalte Flüssigkeiten scheinen als auslösende Schädlichkeit obenan zu stehen; sodann: Aufregung. Aerger, Abspannung. Die Druckempfindlichkeit fehlt oft oder steht doch zu der Heftigkeit des Anfalls in gar keinem Verhältnis. Wiederholte Untersuchungen des Inhalts, der Motilität etc. ergaben inımer negative oder doch nur sehr vieldeutige Resultate. Auch die Magenattacken pflegen sich vielfach an bestimmte Tageszeiten zu halten, ohne daß eine rechte Beziehung zu den Nahrungsaufnahmen erkennbar würde. Recht häufig traten sie nachts auf, namentlich gegen Morgen, eine Zeit, die überhaupt für die Symptome der Rückfallsgrippe eine besondere Bedeutung zu haben scheint: auch neuralgiforme, akralgische und andere Beschwerden stellen sich oft im zweiten Teile der Nacht mit auffallender Heftigkeit ein.

Rheumatische Schmerzformen in Gelenken und Muskelgruppen sind bei der Rückfallsgrippe sehr verbreitet, sei es für sich, sei es mit akralgischen und neuralgischen nıannigfach kombiniert. Auch bei ihnen ist die Neigung zum blitzschnellen Wechsel des Sitzes nach mehrstündiger, -tägiger oder -wöchiger Dauer im Gegensatz zu der örtlichen Hartnäckigkeit der idiopathischen Muskelrheumatismen charakteristisch.

Eigentlicher Kopfschmerz wurde nie beobachtet; entweder handelte es sich um neuralgische Klagen oder un eine Art Benommenheit im Kopfe, geistige Hemmung u. dgl.

Sehr verbreitet ist Herzschmerz; meistens in der auch von den ,Herzneurosen" her bekannten Form eines punktförmigen, brennenden, bohrenden Schmerzes im Bereiche del Herzdämpfung. Ausstrahlen in den linken Arm habe ich nie beobachtet. Manchmal besteht auch ein leises, immer sehr unangenehmes, weil beständig beängstigendes Wehgefühl an ganzen Herzen, das sich namentlich bei Erregung, nach schon ganz kleinen Alkohol- oder Kaffeegenüssen, bei vollem Magen, Flatuswanderung und bei körperlicher Anstrengung (Treppensteigen) vermehrt.

Halsschmerz tritt namentlich in einer charakteristischen Form auf: als neuralgiformes Reißen, Schießen, Brennen in der Schlundgegend. Schluckweh ist häufig. Auch neuralgiforme Zahnschmerzen werden oft beobachtet.

Ueber vage, nicht recht lokalisierbare ,innere" Schmerzen, ähnlich denen, die im Anfange zirkulärer Depressionen oft auftreten und lange Zeit hindurch körperliche Krankheit vortäuschen können, wird viel geklagt. Auch Ermüdungsschmerzen, den Wachstumsschmerzen der Jugend am meisten verwandt, werden schon nach leichten Anstrengungen angegeben. (Schluß folgt.) 